\title{
Optimization of training periods for the estimation model of three-dimensional target positions using an external respiratory surrogate
}

Hiraku Iramina ${ }^{1,2}$, Mitsuhiro Nakamura ${ }^{2,3^{*}}$, Yusuke lizuka², Takamasa Mitsuyoshi², Yukinori Matsuo², Takashi Mizowaki ${ }^{2}$ and Ikuo Kanno ${ }^{1}$

\begin{abstract}
Background: During therapeutic beam irradiation, an unvisualized three-dimensional (3D) target position should be estimated using an external surrogate with an estimation model. Training periods for the developed model with no additional imaging during beam irradiation were optimized using clinical data.

Methods: Dual-source 4D-CBCT projection data for 20 lung cancer patients were used for validation. Each patient underwent one to three scans. The actual target positions of each scan were equally divided into two equal parts: one for the modeling and the other for the validating session. A quadratic target position estimation equation was constructed during the modeling session. Various training periods for the session-i.e., modeling periods $\left(T_{\mathrm{M}}\right)$ - were employed: $T_{M} \in\{5,10,15,25,35\}[s]$. First, the equation was used to estimate target positions in the validating session of the same scan (intra-scan estimations). Second, the equation was then used to estimate target positions in the validating session of another temporally different scan (inter-scan estimations). The baseline drift of the surrogate and target between scans was corrected. Various training periods for the baseline drift correction-i.e., correction periods $\left(T_{C} S\right)$ - were employed: $T_{C} \in\left\{5,10,15 ; T_{C} \leq T_{M}\right\}[s]$. Evaluations were conducted with and without the correction. The difference between the actual and estimated target positions was evaluated by the root-mean-square error (RMSE).
\end{abstract}

Results: The range of mean respiratory period and 3D motion amplitude of the target was 2.4-13.0 s and 2.8-34.2 mm, respectively. On intra-scan estimation, the median 3D RMSE was within 1.5-2.1 mm, supported by previous studies. On inter-scan estimation, median elapsed time between scans was $10.1 \mathrm{~min}$. All $T_{\mathrm{M}}$ exhibited 75th percentile 3D RMSEs of 5. 0-6.4 mm due to baseline drift of the surrogate and the target. After the correction, those for each $T_{\mathrm{M} S}$ fell by 1.4-2. $3 \mathrm{~mm}$. The median 3D RMSE for both the 10-s $T_{\mathrm{M}}$ and the $T_{C}$ period was $2.4 \mathrm{~mm}$, which plateaued when the two training periods exceeded $10 \mathrm{~s}$.

Conclusions: A widely-applicable estimation model for the 3D target positions during beam irradiation was developed. The optimal $T_{M}$ and $T_{C}$ for the model were both $10 \mathrm{~s}$, to allow for more than one respiratory cycle.

Trial registration: UMIN000014825. Registered: 11 August 2014.

Keywords: 3D target motion estimation, Baseline drift correction period, CBCT projection, External surrogate, Training period

\footnotetext{
* Correspondence: m_nkmr@kuhp.kyoto-u.ac.jp

${ }^{2}$ Department of Radiation Oncology and Image-applied Therapy, Graduate School of Medicine, Kyoto University, 54 Kawahara-cho, Shogoin, Sakyo-ku, Kyoto 606-8507, Japan

${ }^{3}$ Division of Medical Physics, Department of Information Technology and Medical Engineering, Human Health Sciences, Graduate School of Medicine, Kyoto University, 53 Kawahara-cho, Shogoin, Sakyo-ku, Kyoto 606-8507, Japan Full list of author information is available at the end of the article
}

(c) The Author(s). 2018 Open Access This article is distributed under the terms of the Creative Commons Attribution 4.0 International License (http://creativecommons.org/licenses/by/4.0/), which permits unrestricted use, distribution, and reproduction in any medium, provided you give appropriate credit to the original author(s) and the source, provide a link to the Creative Commons license, and indicate if changes were made. The Creative Commons Public Domain Dedication waiver (http://creativecommons.org/publicdomain/zero/1.0/) applies to the data made available in this article, unless otherwise stated. 


\section{Background}

Real-time detection of a target's position in threedimensional (3D) space is essential to ensure that high-dose radiation is delivered only to the target, where thus is possible with the aid of four-dimensional cone-beam computed tomography (4D-CBCT). However, the temporal resolution of $4 \mathrm{D}-\mathrm{CBCT}$ is too low to precisely monitor target motion [1]. High-precision radiotherapy requires detection of the 3D position at high temporal resolution (sampling rate $>$ $1 \mathrm{~Hz}$ ). Localization of 3D target position during beam irradiation is used for the dose-of-the-day calculations [2], planning target volume verification [3], and real-time tumor tracking [4-6].

Optimal detection of time-resolved 3D position involves the triangulation of two simultaneous two-dimensional (2D) projections using pre-defined camera parameters [7]. Modern radiotherapy treatment machines are configured with a single-source kilovoltage $(\mathrm{kV})$ imaging subsystem perpendicular to the megavoltage (MV) beam and an electronic portal imaging device. Some machines are equipped with orthogonal dual-source $\mathrm{kV}$ imaging subsystems; such machines allow $\mathrm{kV} / \mathrm{MV}$ or $\mathrm{kV} / \mathrm{kV}$ triangulation. However, depending on the extent of multi-leaf collimator motion during beam irradiation, the target on MV images may not be detectable. Detection of 3D target positions using a monoscopic view is now possible with techniques such as $\mathrm{kV}$ intra-fraction monitoring $[8,9]$ and marker-less tumor detection [10-12]. However, these methods are inapplicable if the target or tumor cannot be detected via $\mathrm{kV}$ projections at certain gantry angles.

To estimate target positions in these situations, there is no alternative but to employ external respiratory surrogates. Cho et al. developed a linear internal-external correlation model with frequent model updating during beam irradiation [13-15]. In their studies, the linear model was selected and updated by minimization of the difference between the measured $\mathrm{kV}$ projections and estimated trajectory projected onto a $2 \mathrm{D}$ projection geometry. As mentioned above, however, updating cannot be applied if the target or tumor cannot be detected on $\mathrm{kV}$ projections, as this would lead to model degradation. This problem has more of an impact on marker-less tumor tracking.

Akimoto et al. pointed out that the visibility of gold markers implanted in the lung on $\mathrm{kV}$ projections could be poor in the following situations: (1) overlap of the gold markers and (2) when there is a low-intensity ratio between the gold marker and its surroundings [16]. Bahig et al. investigated the applicability of a marker-less tumor tracking system using CyberKnife (XSight Lung Tracking System) via a fixed dual-source $\mathrm{kV}$ imaging subsystem [17]. They revealed that $50 \%$ of tumors smaller than $2 \mathrm{~cm}$ cannot be visualized on the $\mathrm{kV}$ projection. Thus, a means to evaluate the estimation model without updating during beam irradiation is required, regardless of whether gold markers are implanted.
Fassi et al. developed a target estimation method using a patient-specific breathing model derived a priori from 4D-CT images [18]. Model parameters were retrieved and updated for each treatment fraction according to inroom radiography acquisition and optical surface imaging. However, the low temporal resolution and imaging artifacts associated with 4D-CT may affect the model accuracy and cannot be improved by updating the model at each treatment fraction.

In this study, we developed a method to estimate target positions employing an external surrogate which was an infrared reflective (IR) marker; our approach is widely applicable and does not require additional imaging during beam irradiation. We applied the quadratic polynomial equation used by the Vero system [19]. The equation incorporated data from the internal target, and position and velocity data from the external IR marker placed on the patient's abdomen; data were acquired at a frequency of $5 \mathrm{~Hz}$. To validate the method, we used the dual-source 4D-CBCT projection data of lung cancer patients who underwent stereotactic body radiotherapy (SBRT); data included the actual 3D target positions during the 4D-CBCT scan, calculated from 2D orthogonal projections featuring $\mathrm{kV} / \mathrm{kV}$ triangulation. First, the estimation equation was modeled using the detected 3D target positions and IR marker positions prior to target position estimation in the same scan (intra-scan estimation). Second, the equation was used to estimate target and surrogate positions in the validation session of another scan (inter-scan estimation). The baseline drifts of the target and IR marker between scans were corrected. Thus, the training periods for these sessions were optimized using the data from clinical practice.

\section{Methods}

\section{Patient characteristics}

Twenty-two consecutive lung cancer patients (17 males, 5 females; median age, 81 years; range: 65-90 years) who underwent SBRT by Vero4DRT (Mitsubishi Heavy Industries, Ltd., Hiroshima, Japan, and BrainLAB AG, Feldkirchen, Germany) after implantation of two-to-four 1.5-mm-diameter gold markers (Olympus, Tokyo, Japan) were enrolled in an institutional review board-approved trial. Each patient underwent three 70-s 4D-CBCT scans, except for Patient 17, and Patients 10 and 18, who underwent only one, and two scans, respectively. Three scans were performed for Patients 1 and 2 were employed in this study; the second scan of three scans for the other patients was taken for a different purpose, and with different scan parameters, and was not applied 4D-CBCT image reconstruction. Thus, the first and third scans of the other patients were used for the validation process. Both scans for Patients 10 and 18 were used for the validation. 
A set of three IR markers was placed on each patient to record abdominal motion in the anteriorposterior (AP) direction. IR marker movement was captured using a portable Polaris Spectra camera (Northern Digital Inc., Ontario, Canada) placed close to the patient; the camera operated independently of the Vero4DRT system. Due to unexpected system problems, the IR marker signals from Patients 8 and 12 were not available. Thus, 20 patients were studied.

\section{Data acquisition}

We recorded 351 projection images (70-s scans per source; i.e., the sampling time, $\Delta t=0.2 \mathrm{~s}$ ). All gold markers were simultaneously detected on all projections. The gold marker located nearest the tumor was considered the best surrogate of the target. The 2D positional data of each projection, or each discrete time $t_{k}(k=0,1$, $\cdots, 350[=70 / \Delta t])$, were converted into $3 \mathrm{D}$ data using the pre-defined camera parameters, to yield actual 3D target positions $P_{\text {actual }}\left(t_{k}\right)$. Data acquisition details have been described previously [1].

The respiratory period during a scan was defined as the average interval between two consecutive end inhalations. The respiratory amplitude was defined as the average difference between the end-exhalation and endinhalation positions of a single respiratory cycle.
Target position estimation using the external surrogate

Our proposed estimation method is shown in Fig. 1. We assumed that the target position would be estimated during beam irradiation using an estimation equation modeled before the irradiation. Thus, we considered two sessions: the modeling and validating sessions. To estimate the target positions during the validating session, construction of the estimation equation was necessary during the modeling session. The quadratic polynomial equation used in the proposed method can be written as follows:

$$
\begin{aligned}
P_{i, \gamma}\left(t_{k}\right)= & a_{i, \gamma} w^{2}\left(t_{k}\right)+b_{i, \gamma} w\left(t_{k}\right)+c_{i, \gamma} \\
& +d_{i, \gamma}\left(\frac{\Delta w}{\Delta t}\right)^{2} \\
& +e_{i, \gamma}\left(\frac{\Delta w}{\Delta t}\right) \equiv \mathbf{C}_{i, \gamma} \cdot \mathbf{W}\left(t_{k}\right) .
\end{aligned}
$$

$\mathbf{C}_{i, \gamma}=\left(a_{i, \gamma} b_{i, \gamma} c_{i, \gamma} d_{i, \gamma} e_{i, \gamma}\right)$ is the coefficient of the equation for IR marker number $i \in\{1,2,3\}$ in direction $\gamma \in\{$ left - right (LR), superior - inferior (SI), anterior - pos-

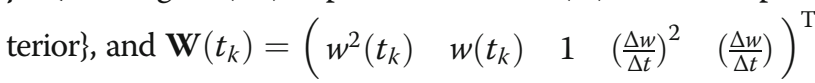
is the IR marker data set at the discrete time $t_{k}$, containing the IR marker position $w\left(t_{k}\right)$ and its derivative-i.e., velocity, $\Delta w / \Delta t$-where $\quad \Delta w=w\left(t_{k}\right)-w\left(t_{k-1}\right) \quad$ and $\Delta t=t_{k}-t_{k-1}=0$.

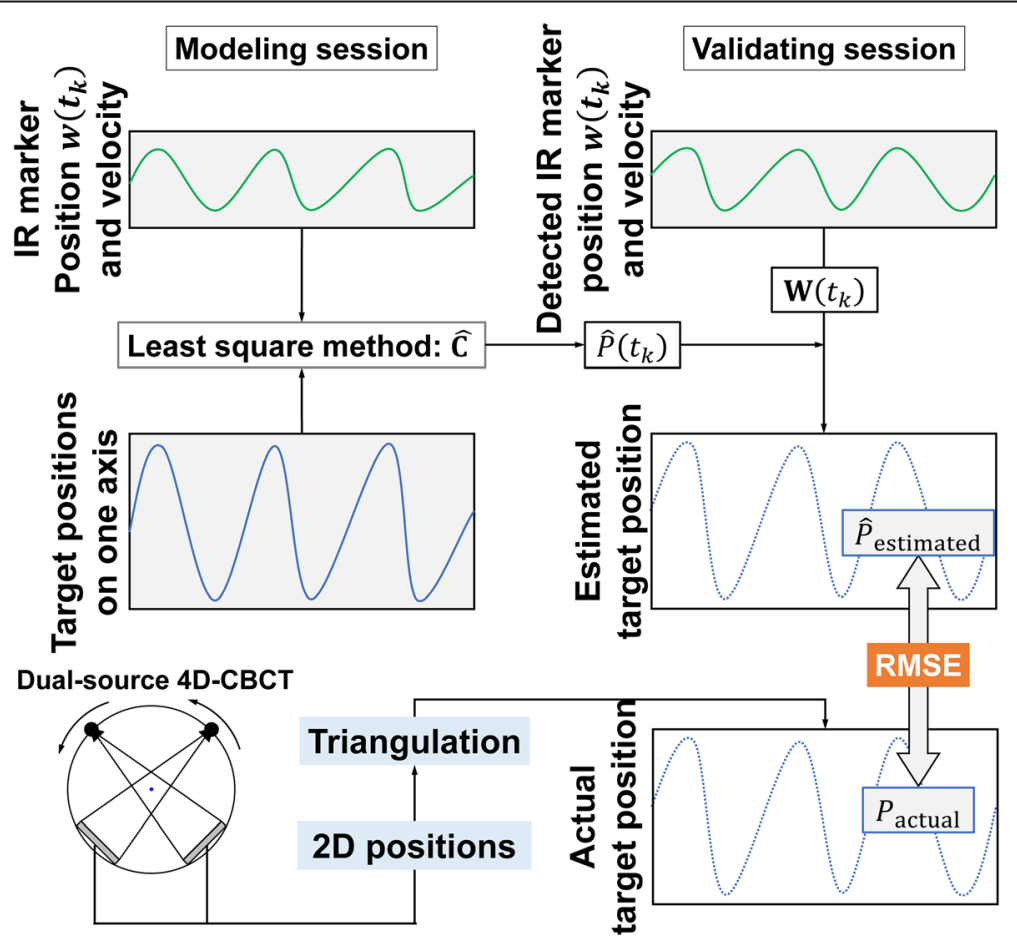

Fig. 1 Flow and validation of our method. The estimation equation, $P\left(t_{k}\right)$, is constructed during the modeling session. $t_{k}$ is the discrete time. The

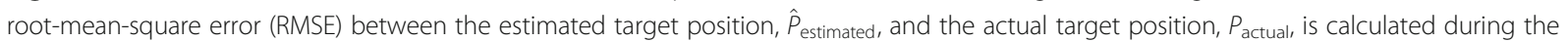
validation session. C: coefficients for $P\left(t_{k}\right)$. W $\left(t_{k}\right)$ : the position and velocity of the infrared reflective (IR) marker at $t_{k}$. 2D: two-dimensional 
2 [s]. Because the following methodology is the same for any IR marker number and direction, we eliminated $i$ and $\gamma$ for simplicity. We divided each of the 351 projections into a first (175 projections, approximately $35 \mathrm{~s}$ ) and second (176 projections, approximately $35 \mathrm{~s}$ ) half for modeling and validating sessions, respectively.

First, we determined the coefficients of the estimation equation during the modeling session and then estimated the target positions during the validating session of the same scan; i.e., an intra-scan estimation. To construct the estimation equation, coefficient $\mathbf{C}$ was determined by the least-squares method using the data of the modeling session. We employed various training periods for modeling-i.e., the modeling period $T_{\mathrm{M}} \in$ $\{5,10,15,25,35\}$ [s] -as shown in Fig. 2a. Thus, a coefficient was determined for each $T_{\mathrm{M}}$ and written as:

$$
\hat{\mathbf{C}}_{T_{\mathrm{M}}}=\operatorname{argmin} \sum_{k=\left(35-T_{\mathrm{M}}\right) / \Delta t+1}^{35 / \Delta t}\left\|P_{\text {actual }}\left(t_{k}\right)-\mathbf{C} \cdot \mathbf{W}\left(t_{k}\right)\right\|^{2} .
$$

Note that the data at $t_{\left(35-T_{\mathrm{M}}\right) / \Delta t}$ were also used because the velocity of the IR marker was necessary. Hereinafter, we use the hat symbol for coefficients determined by the least-squares method, and the estimation equation that included the coefficients, or $\hat{P}_{T_{M}}\left(t_{k}\right)$. The target positions within the validating session of the same scan were estimated using the estimation equation in each direction.

Second, we estimated the target positions in the validating session using the estimation equation constructed during the modeling session of another scan in a one patient; i.e., an inter-scan estimation (Fig. 2b). In clinical cases, the baseline drift correction will be applied between fields or arcs if multiple beams are used in the plan. Let $\hat{P}_{T_{\mathrm{M}}}^{\mathrm{A} \rightarrow \mathrm{B}}\left(t_{k}\right)$ be the estimation equation for the target positions of scan $B$ by using the coefficients determined by the data of scan A. Due to the elapsed time between the two scans, the baseline drift of the IR marker and target should be corrected. The baseline of scan $\mathrm{A}$ is defined as the median position in the training period for the baseline drift correction-i.e., the correction period $T_{\mathrm{C}} \in\left\{5,10,15 ; T_{\mathrm{C}} \leq T_{\mathrm{M}}\right\}$ [s]-at the end of the modeling session or prior to the validating session (Fig. 2b). The baseline of scan B is defined as the median position in the correction period $T_{\mathrm{C}}$ immediately prior to the validating session of scan $B$. The amount of the baseline drift between scans $A$ and $B$ was calculated by subtracting the baseline of scan A from that of scan B. First, we corrected the baseline drift of the IR marker. The amount of baseline drift of the IR marker, defined as follows:

$$
\begin{aligned}
\mathrm{BD}_{\mathrm{IR}, T_{\mathrm{C}}}^{\mathrm{B}-\mathrm{A}}= & \operatorname{median}\left\{w^{\mathrm{B}}\left(t_{k}\right), k \in\left\{\frac{35-T_{\mathrm{C}}}{\Delta t}: \frac{35}{\Delta t}\right\}\right\} \\
& - \text { median }\left\{w^{\mathrm{A}}\left(t_{k}\right), k \in\left\{\frac{35-T_{\mathrm{C}}}{\Delta t}: \frac{35}{\Delta t}\right\}\right\} .
\end{aligned}
$$

Then, we incorporated the baseline drift of IR marker by transforming $w^{\mathrm{B}}\left(t_{k}\right)$ into $w^{\mathrm{B}}\left(t_{k}\right)-\mathrm{BD}_{\mathrm{IR}, T_{\mathrm{C}}}^{\mathrm{B}-\mathrm{A}}$ and let $\left[\hat{P}_{\left(T_{\mathrm{M}}, T_{\mathrm{C}}\right)}^{\mathrm{A} \rightarrow \mathrm{B}}\left(t_{k}\right)\right]^{\prime}$ be the estimation equation $\hat{P}_{T_{\mathrm{M}}}^{\mathrm{A} \rightarrow \mathrm{B}}\left(t_{k}\right)$ scaled by $\mathrm{BD}_{\mathrm{IR}, T_{\mathrm{C}}}^{\mathrm{B}-\mathrm{A}}$. To incorporate the baseline drift of the target, we compensated for internal residual errors resulting from the baseline drift of $\left[\hat{P}_{T_{\mathrm{M}}}^{\mathrm{A} \rightarrow \mathrm{B}}\left(t_{k}\right)\right]^{\prime}$-i.e., $\mathrm{BD}_{\text {Target, } T_{\mathrm{C}}}^{\mathrm{B}-\mathrm{A}}$ - which can be written as

$$
\begin{aligned}
\mathrm{BD}_{\text {Target }, T_{\mathrm{C}}}^{\mathrm{B}-\mathrm{A}}= & \operatorname{median}\left\{\left[\hat{P}_{\left(T_{\mathrm{M}}, T_{\mathrm{C}}\right)}^{\mathrm{A} \rightarrow \mathrm{B}}\left(t_{k}\right)\right]^{\prime}, k \in\left\{\frac{35-T_{\mathrm{C}}}{\Delta t}: \frac{35}{\Delta t}\right\}\right\} \\
& - \text { median }\left\{P_{\text {actual }}^{\mathrm{A}}\left(t_{k}\right), k \in\left\{\frac{35-T_{\mathrm{C}}}{\Delta t}: \frac{35}{\Delta t}\right\}\right\} .
\end{aligned}
$$

Then, we subtracted $\mathrm{BD}_{\text {Target }, T_{\mathrm{C}}}^{\mathrm{B}-\mathrm{A}}$ from $\left[\hat{P}_{\left(T_{\mathrm{M}}, T_{\mathrm{C}}\right)}^{\mathrm{A} \rightarrow \mathrm{B}}\left(t_{k}\right)\right]^{\prime}$, and let $\left[\hat{P}_{\left(T_{\mathrm{M}}, T_{\mathrm{C}}\right)}^{\mathrm{A} \rightarrow \mathrm{B}}\left(t_{k}\right)\right]^{\mathrm{Cor}}$ be the baseline drift-corrected estimation equation. To increase the number of data sets, we estimated the target positions in the validating session of scan A using the estimation equation constructed in the modeling session of scan $\mathrm{B}$. The relationship between the intra- and inter-scan estimations is shown in Fig. 2c. The 4D-CBCT projection data for Patient 17 were only used to validate the intra-scan estimation, because the patient underwent 4D-CBCT only once.

For comparison, we also used a $T_{\mathrm{M}}$ of $70 \mathrm{~s}$. Note that the validating session was included to model the estimation equation $\hat{P}_{T_{\mathrm{M}}=70}\left(t_{k}\right)$ for the intra-scan estimation. For the inter-scan estimation, the baseline drifts of the IR marker and target were calculated, and correction was carried out using the process described above.

The linear estimation equation constructed from eight 4D-CBCT positions was also utilized. Eight averaged target positions for each phase, $P_{4 \mathrm{D}-\mathrm{CBCT}}(l)$ $(l \in\{0,1, \cdots, 7\})$, were obtained from one 4D-CBCT scan. In addition, eight averaged IR marker positions, $w_{\text {ave }}(l)(l \in\{0,1, \cdots, 7\})$, were obtained for each phase. We defined phases 0 and 4 as end-exhalation and end-inhalation, respectively. The linear estimation equation was constructed between each phase; between phase $\alpha$ and $\beta$, it was written as follows:

$$
\hat{P}_{\text {Linear }}^{(\alpha, \beta)}\left(t_{k}\right)=a^{(\alpha, \beta)} w\left(t_{k}\right)+b^{(\alpha, \beta)}
$$



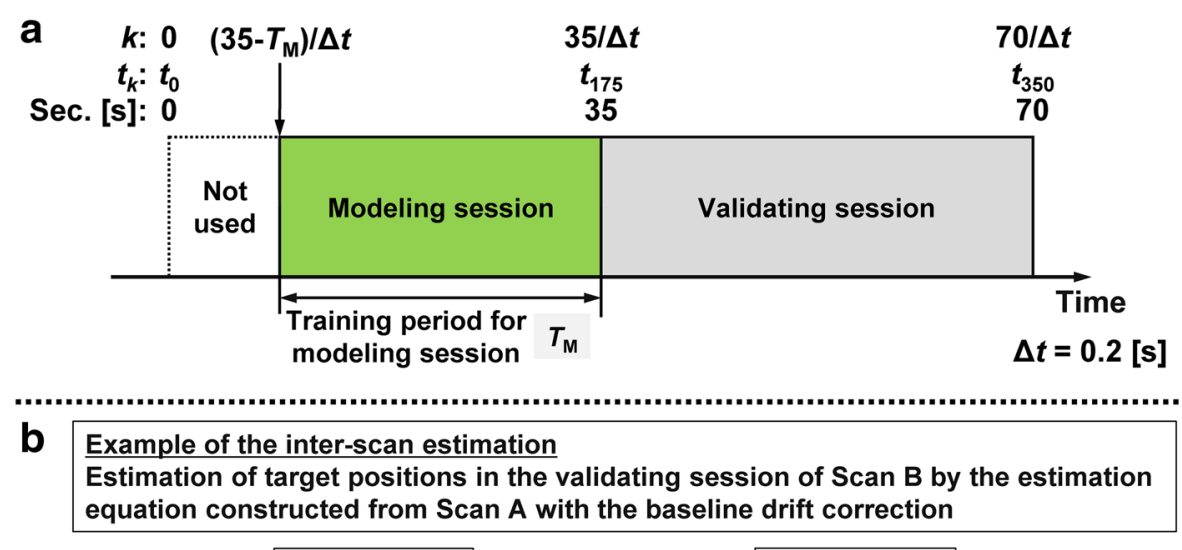

CBCT Scan A CBCT Scan B

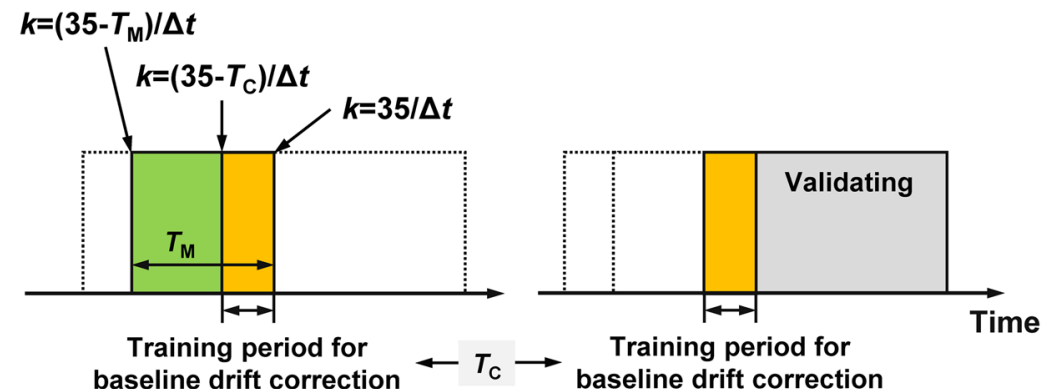

C

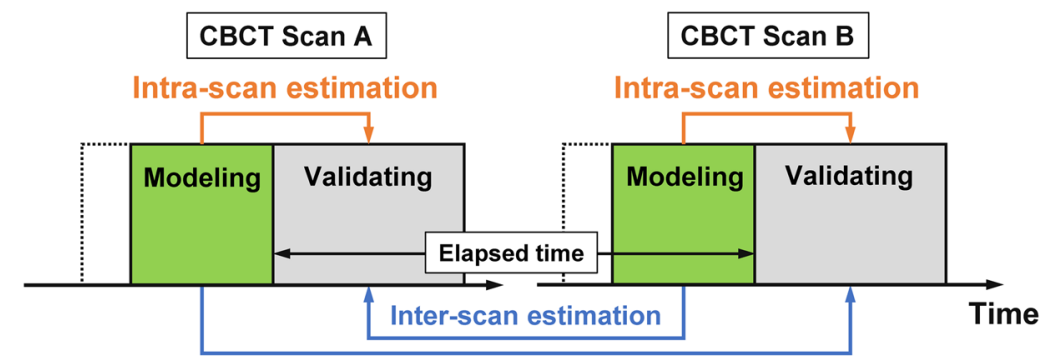

Inter-scan estimation

Fig. 2 a Details of the training period for the modeling session $\left(T_{M}\right) ; \Delta t$ is the sampling rate. $\mathbf{b}$ Example of an inter-scan estimation. The relationship between $T_{M}$ and the training period for the baseline drift correction $\left(T_{C}\right)$ is shown. $\left[\hat{P}_{\left(T_{M}, T_{C}\right)}^{A \rightarrow B}\left(t_{k}\right)\right]^{C o r}$ is the baseline drift-corrected estimation equation. c The relationship between intra- and inter-scan estimations on two cone-beam computed tomography (CBCT) scans

$$
\text { if } w_{\text {ave }}(\alpha) \leq w\left(t_{k}\right) \leq w_{\text {ave }}(\beta),(\alpha, \beta) \in\{(0,1),(1,2), \cdots,(7,0)\}
$$

In the validating session, the IR marker position $w\left(t_{k}\right)$ was distinguished as mid-exhalation and mid-inhalation when $\Delta w / \Delta t>0$ and $\Delta w / \Delta t<0$, respectively. In the inter-scan estimation, the centroid position of the eight averaged target positions was recognized as the baseline for a $4 \mathrm{D}-\mathrm{CBCT}$ scan. The baseline drift correction for Eq. (5) was employed similarly as mentioned above. The number of internal residual errors resulting from the baseline drift of $\left[\hat{P}_{\text {Linear }}^{(\alpha, \beta)}\left(t_{k}\right)\right]^{\prime}$ is given by

$$
\begin{aligned}
& \mathrm{BD}_{\text {Target, }\left(T_{\mathrm{C}, 4 \mathrm{D}-\mathrm{CBCT}}\right)}^{\mathrm{B}-\mathrm{A}}=\operatorname{median}\left\{\left[\hat{P}_{\text {Linear }}^{(\alpha, \beta)}\left(t_{k}\right)\right]^{\prime}, k \in\left\{\frac{35-T_{\mathrm{C}}}{\Delta t}: \frac{35}{\Delta t}\right\}\right\} \\
& -\frac{1}{8} \sum_{l=0}^{7} P_{4 \mathrm{D}-\mathrm{CBCT}}^{\mathrm{A}}(l) \text {. }
\end{aligned}
$$

Because the estimation equation is constructed for each IR marker $i$, the estimated target position is defined as the average of the target position estimated from each IR marker, written as follows: $\hat{P}_{\text {estimated }}\left(t_{k}\right)$ $=\frac{1}{3} \sum_{i=1}^{3} \hat{P}_{i}\left(t_{k}\right)$. The difference between the estimated target position $\hat{P}_{\text {estimated }}\left(t_{k}\right)$ and actual target position $P_{\text {actual }}\left(t_{k}\right)$ in each direction was evaluated in terms of the root-mean-square error (RMSE). 


\section{Statistical analysis}

We tested for equality of variance prior to performing multiple pairwise comparisons. Depending on the equality of variance status, we used either oneway analysis of variance or the non-parametric Kruskal-Wallis test to compare the differences. If a difference was significant, the non-parametric SteelDwass test was performed subsequently to simultaneously evaluate all differences between training periods. A $p$-value $<0.05$ was considered statistically significant.

\section{Results}

\section{Respiratory patterns}

The range of mean period was 2.4-13.0 s; the range of mean IR marker amplitude in the AP direction was $1.7-14.6 \mathrm{~mm}$; and the range of mean target amplitude in the LR, SI, AP directions, and 3D motion were $0.3-11.7,1.4-28.7,0.7-18.1$, and $2.8-34.2 \mathrm{~mm}$, respectively. The respiratory amplitudes during the various scan periods of all patients, and the correlation coefficients between IR marker motion along the AP direction and target motions along the LR, SI, and AP directions, are shown in Fig. 3; large error bars indicate irregular breathing, such as apnea, tachypnea, hypopnea, hyperpnea, or combinations of these. The target motion in the SI direction was strongly positively correlated with IR marker motion.

\section{Intra-scan estimations}

The RMSEs in the LR, SI, AP, and 3D directions of the intra-scan estimations are shown in Fig. 4. The RMSEs in the SI direction were associated with larger errors than those of the other directions. The median RMSEs in the LR and AP directions were $<1.0 \mathrm{~mm}$. The median 3D RMSEs for all $T_{\mathrm{M}} \mathrm{s}$ were $1.5-2.1 \mathrm{~mm}$ and gradually declined as $T_{\mathrm{M}}$ became longer. Although the KruskalWallis test revealed significant differences among $T_{\mathrm{M}} \mathrm{S}$ values in the LR direction $(p<0.05)$, the 3D RMSEs did not differ significantly. However, the $T_{\mathrm{M}} \mathrm{S}$ of $5 \mathrm{~s}$-i.e., $T_{\mathrm{M}}=5 \mathrm{~s}$-showed large outliers in all directions, and the 3D RMSE of Patient 10 resulted in large outliers in $T_{\mathrm{M}}=5,10,15,25$, and $35 \mathrm{~s}$. Note that although the estimation equation modeled by $T_{\mathrm{M}}=4 \mathrm{D}-\mathrm{CBCT}$ and $T_{\mathrm{M}}=70 \mathrm{~s}$ showed small errors, these $T_{\mathrm{M}} \mathrm{s}$ had included $P_{\text {actual }}$ to construct the linear and quadratic estimation equations, respectively.

\section{Inter-scan estimations}

The median elapsed time between scans was $10.1 \mathrm{~min}$ (range: 1.7-15.0 $\mathrm{min}$ ). The absolute baseline drift of an IR marker, which was calculated during each baseline drift $T_{\mathrm{C}}$ is shown in Fig. 5. The median absolute baseline drift for $T_{\mathrm{C}}=5,10$, and $15 \mathrm{~s}$ was $1.5 \mathrm{~mm}$ (range: $0.1-9.6 \mathrm{~mm}$ ), $0.8 \mathrm{~mm}$ (range: $0.0-5.0 \mathrm{~mm}$ ), and $1.2 \mathrm{~mm}$ (range: $0.0-5.5 \mathrm{~mm}$ ), respectively. No significant difference among each $T_{\mathrm{C}}$ values was found. The time series of the 3D RMSEs in $T_{\mathrm{C}}$ for each $T_{M}$ is shown in Fig. 6. Although 3D RMSEs for all $T_{\mathrm{M}} \mathrm{s}$ exhibited large values without the baseline drift correction, they were decreased drastically by the correction, except for the 4D-CBCT data.

Figure 7 shows $P_{\text {actual }}$ and $\hat{P}_{\text {estimated }}$ without and with baseline drift correction of the first scan of $\mathrm{Pa}$ tient 2 , determined using the estimation equations $\hat{P}_{T_{M}=10}^{3 \mathrm{rd} \rightarrow 1 \mathrm{st}}\left(t_{k}\right)$ and $\left[\hat{P}_{\left(T_{\mathrm{M}}=10, T_{\mathrm{C}}=10\right)}^{3 \mathrm{rd} \rightarrow 1 \mathrm{~s}}\left(t_{k}\right)\right]^{\text {Cor }}$, respectively; both the $T_{\mathrm{M}}$ and the $T_{\mathrm{C}}$ was $10 \mathrm{~s}$. In this case, 3D RMSE was reduced from $5.4 \mathrm{~mm}$ to $1.1 \mathrm{~mm}$ with the baseline drift correction. In contrast, in several cases the 3D RMSE was over $6 \mathrm{~mm}$ even with the baseline drift corrections, due to a sudden change in the correlation between the IR marker and the target in the validating session in Fig. 6. Once again, the $T_{\mathrm{M}}=5 \mathrm{~s}$ data exhibited large outliers in all directions. Moreover, the 3D RMSE of Patient 10 resulted in severe outliers in all $T_{\mathrm{M}} \mathrm{S}$, except for the $T_{\mathrm{M}}=$ 4D-CBCT data.

Boxplots of the 3D RMSEs are shown in Fig. 8. The 75th percentile 3D RMSEs without baseline drift correction were $5.0-6.4 \mathrm{~mm}$. After baseline drift correction, the 75th percentile 3D RMSEs for each $T_{\mathrm{M}}$ s fell by $1.4-2.3 \mathrm{~mm}$, except for the $T_{\mathrm{M}}=4 \mathrm{D}-\mathrm{CBCT}$, which increased by $0.6-1.2 \mathrm{~mm}$. Prolongation of both $T_{\mathrm{M}}$ and $T_{\mathrm{C}}$ reduced the median 3D RSMEs; however, this effect plateaued at $T_{\mathrm{M}} \mathrm{S}$ and $T_{\mathrm{C}} \mathrm{S}>10 \mathrm{~s}$. The SteelDwass test revealed significant differences between $T_{\mathrm{M}}=4 \mathrm{D}-\mathrm{CBCT}$ with baseline drift correction and several $T_{\mathrm{M}} \mathrm{S}($ all $p<0.05)$.

\section{Discussion}

Similar to other studies [20,21], the patients enrolled in this study exhibited various combinations of respiratory period and amplitude; the mean respiratory period was approximately $4 \mathrm{~s}$. Target motion along SI direction had strong correlation with IR marker motion along the AP direction, as also found by Ionascu et al. [22]. Several target motions correlated only weakly with IR marker motion because breathing was sometimes irregular, as shown by the large error bars on the amplitude axis in Fig. 3a-3d. As target positions were estimated using only the IR marker, the weak correlations between target and IR marker positions created large RMSEs [23].

Following intra-scan estimations, the median RMSEs for the estimated target positions-i.e., -in the LR, AP, and SI directions were 1-2 $\mathrm{mm}$, as also found by Ruan et al. [24]. Although the 75th percentile 3D RMSE for the $T_{\mathrm{M}}$ of $5 \mathrm{~s}$ was $<4 \mathrm{~mm}$ in most patients, several large 

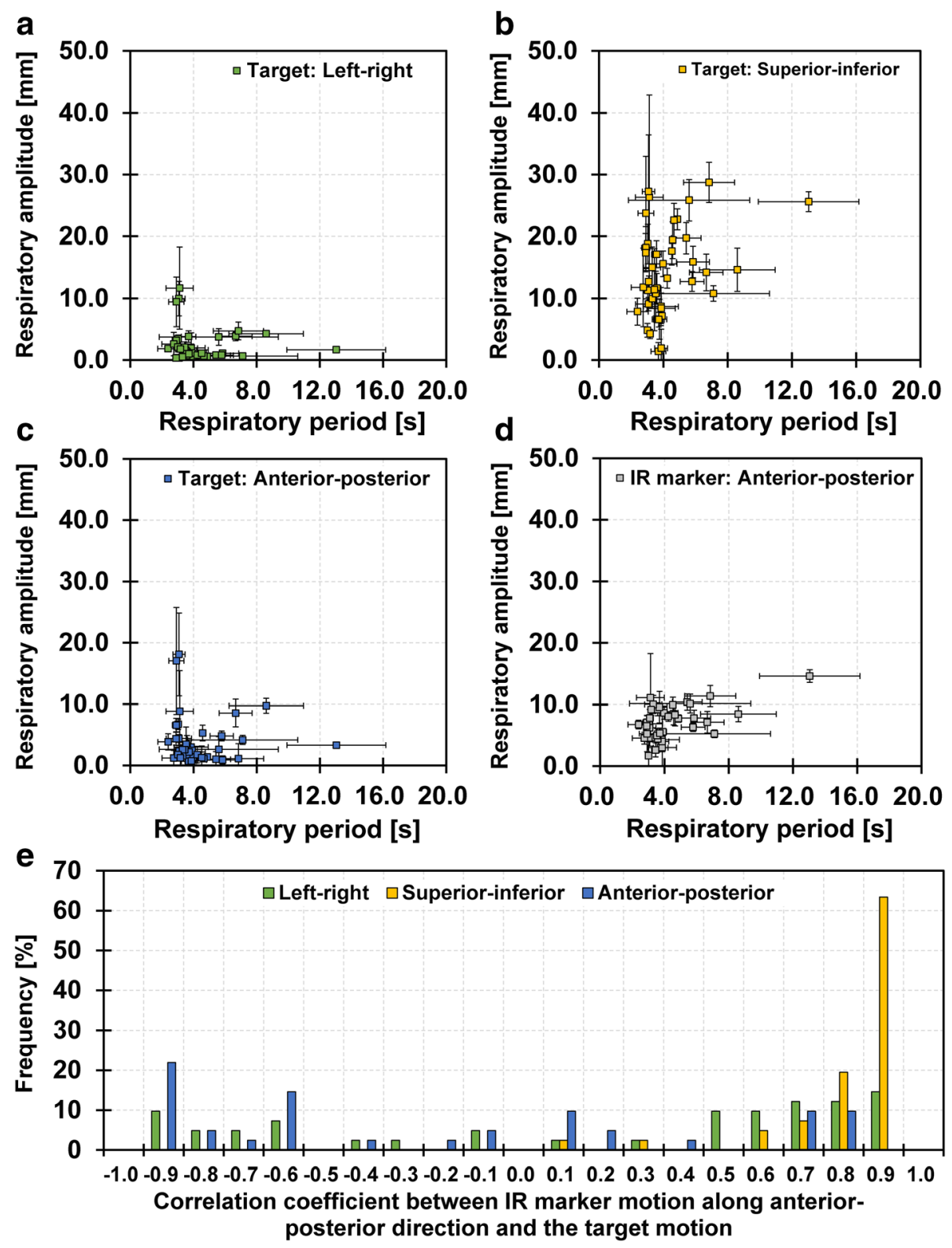

Fig. 3 Scatter plots of the respiratory periods of all patients, and the target motion amplitude in (a) the left-right, (b) the superior-inferior, and (c) the anterior-posterior (AP) directions; and (d) that of the infrared reflective (IR) marker in the AP direction. (e) Correlation coefficients between IR marker motion in the AP direction and target motion

RMSEs were observed. The proportion of patients with a respiratory period within 5 and $10 \mathrm{~s}$ was 78 and $98 \%$, respectively. Thus, $T_{M}$ must cover at least one respiratory cycle, which was set to $10 \mathrm{~s}$ in this study. The median 3D RMSEs fell as the $T_{M}$ was prolonged. The median 3D RMSE for $T_{\mathrm{M}}=10 \mathrm{~s}$ and $T_{\mathrm{M}}=35 \mathrm{~s}$ was 2.1 and $2.0 \mathrm{~mm}$, respectively. No significant difference was apparent among the RMSEs for each $T_{\mathrm{M}}$. Therefore, a training period of $10 \mathrm{~s}$ would be appropriate to model the estimation equation. In comparison, $T_{\mathrm{M}}=70$ showed a median 3D RMSE of $1.7 \mathrm{~mm}$.
Cho et al. developed a method in which the 3D target position could be estimated using a linear equation that included the position of the external surrogate, and thus requires frequent model updating was required during beam irradiation [13]. Forty-six thoracic and abdominal cancer patients were investigated in their study and they calculated that updates at $1 \mathrm{~Hz}$ yielded 3D RMSEs of nearly $1 \mathrm{~mm}$, similar to the RMSEs of stereoscopic estimations. However, the range in the mean $3 \mathrm{D}$ motion of the targets located at the left or right lower lobe of the lungs was $0.2-$ 


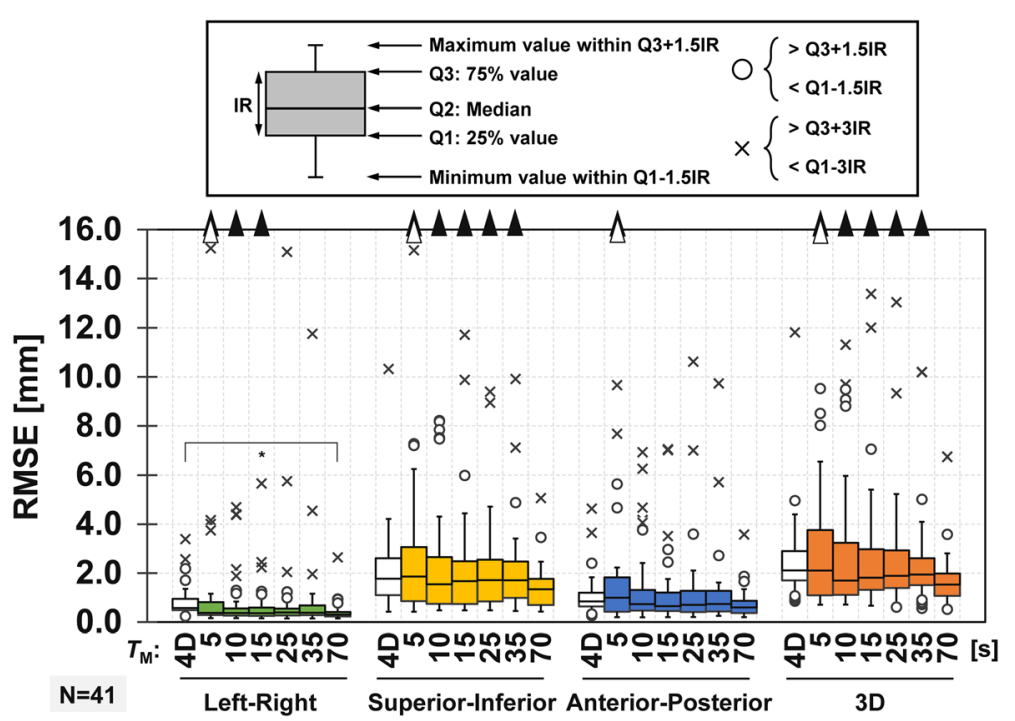

Fig. 4 Boxplots of RMSEs for each training period within a modeling session (the $T_{M}$ values) in the left-right, superior-inferior, anterior-posterior, and three-dimensional (3D) directions, calculated via intra-scan estimations. White triangles: outliers out of range. Black triangles: outliers observed in patient included apnea and hyperpnea data. ${ }^{*} p<0.05$

$14.4 \mathrm{~mm}$ in the study $[13-15,25]$, whereas in our study we observed a range of 3.7-28.4 mm. For instance, excluding the data that showed a 3D target amplitude larger than $14.4 \mathrm{~mm}$, the 3D RMSE for $T_{\mathrm{M}}=10 \mathrm{~s}$ was $1.1 \mathrm{~mm}$ (data shown in Additional file 1). As mentioned above, updating is impossible if the $\mathrm{kV}$ image is temporarily unavailable, which would lead to degradation of the model and greater impact on marker-less tumor tracking. Again, the implanted gold markers were not always visible in situations with marker overlap and low marker contrast relative to the surroundings. Moreover, the model developed by Fassi et al. required no additional imaging during beam irradiation [18]. Although the cited authors evaluated the estimation errors from 2D positions on CBCT, which were projected from estimated 3D positions, the errors were about $2 \mathrm{~mm}$. One advantage of our method is that MV and kV images are not required during beam irradiation; additionally, the RMSE associated with our method is comparable to those of previous study [13].

Regarding inter-scan estimations, the estimation equations were applied to other scans, and optimal $T_{\mathrm{M}}$ and $T_{\mathrm{C}}$ values were determined. The 3D RMSEs for the $T_{\mathrm{C}}$ of each $T_{\mathrm{M}}$ fell when the baseline drift corrections were applied, except in the case of $T_{\mathrm{M}}=$ 4D-CBCT. Twenty-nine of forty-six inter-scan estimations for $T_{\mathrm{M}}=4 \mathrm{D}-\mathrm{CBC}$ estimations exhibited no

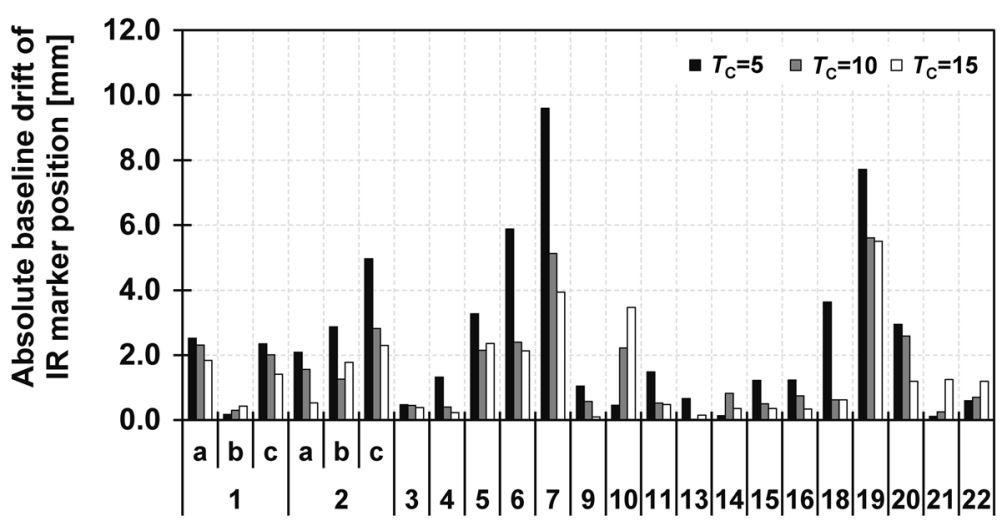

Patient \#

Fig. 5 Absolute baseline drift of the IR marker, which calculated during each baseline drift correction period $\left(T_{C}=5,10\right.$, and $\left.15 \mathrm{~s}\right)$. In the graph, $a, b$, and $c$ for Patients 1 and 2 indicate the baseline drift between first and second scans, first and third scans, and second and third scans, respectively 

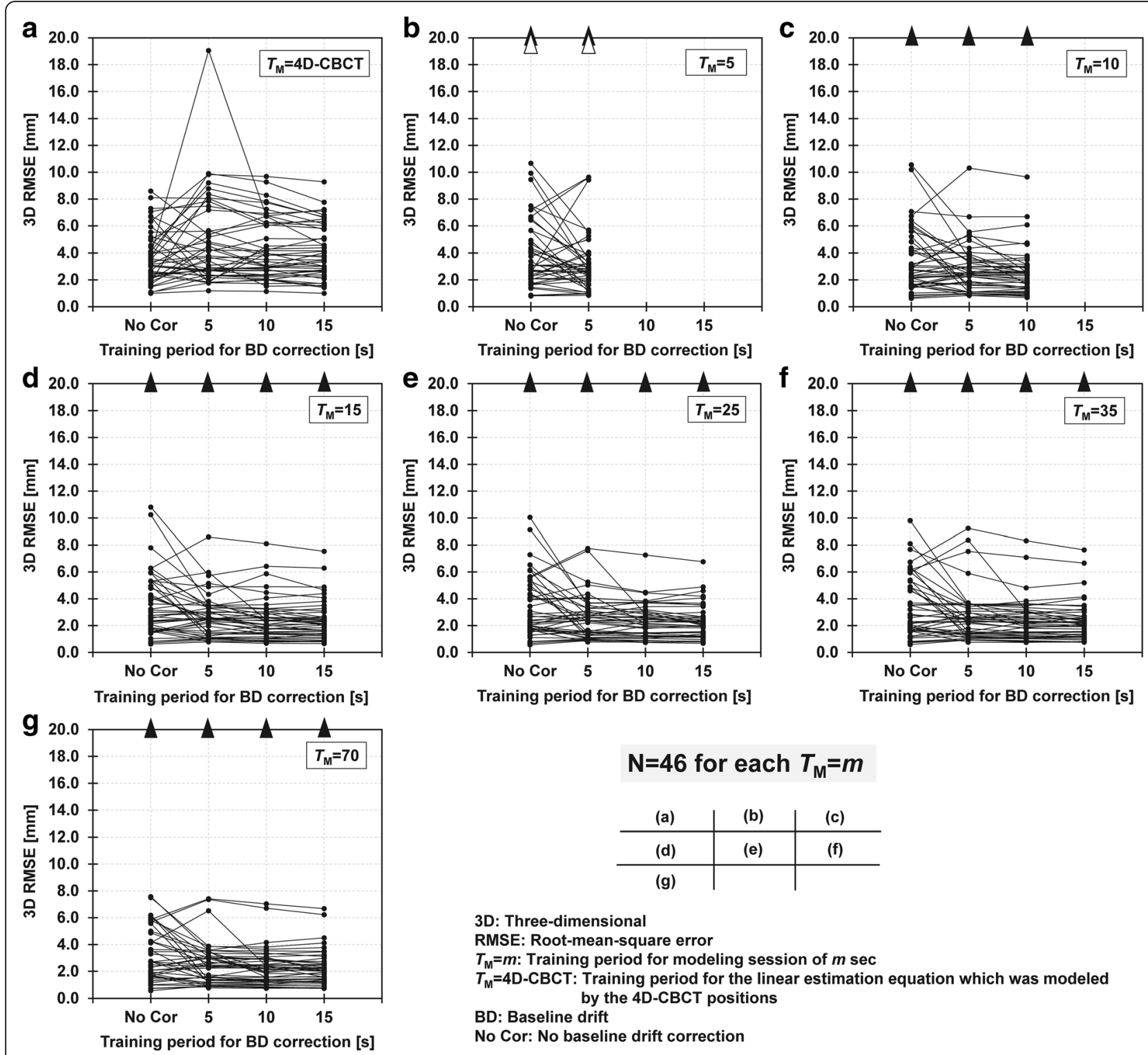

\begin{tabular}{l|l|l} 
(a) & (b) & (c) \\
\hline (d) & (e) & (f) \\
\hline (g) & &
\end{tabular}

3D: Three-dimensional

RMSE: Root-mean-square error

$T_{\mathrm{M}}=m$ : Training period for modeling session of $\boldsymbol{m} \mathrm{sec}$

$T_{\mathrm{M}}=4 \mathrm{D}-\mathrm{CBCT}$ : Training period for the linear estimation equation which was modeled

BD: Baseline drift by the 4D-CBCT positions

No Cor: No baseline drift correction

Fig. 6 Changes in 3D RMSEs due to differences in the correction period for the modeling period of (a) four-dimensional cone-beam computed tomography, (b) $5 \mathrm{~s},(\mathbf{c}) 10 \mathrm{~s},(\mathbf{d}) 15 \mathrm{~s},(\mathbf{e}) 25 \mathrm{~s},(\mathbf{f}) 35 \mathrm{~s}$, and (g) $70 \mathrm{~s}$. White triangles: outliers out of range. Black triangles: outliers observed in patient included apnea and hyperpnea data

improvement upon baseline drift correction. The 3D RMSEs for corrected $T_{\mathrm{M}}=4 \mathrm{D}-\mathrm{CBCT}$ values showed a larger interquartile range and longer error bars than did those without the correction, as well as the 3D RMSEs of all other $T_{\mathrm{M}} \mathrm{s}$ (Fig. 8). This is because the power of the linear estimation equation is first-order in nature, and thus is insensitive to baseline drift correction. On the other hand, the 3D RMSEs of other $T_{\mathrm{M}} \mathrm{S}$ after correction exhibited small interquartile ranges and lower error bars. The median 3D RMSE fell as $T_{\mathrm{M}}$ and $T_{\mathrm{C}}$ were prolonged, plateauing at $T_{\mathrm{M}}=10 \mathrm{~s}$ for each $T_{\mathrm{C}}$. The minimum median 3D RMSE was $2.0 \mathrm{~mm}$ when $T_{\mathrm{M}}=70 \mathrm{~s}$ and $T_{\mathrm{C}}=15 \mathrm{~s}$ were combined; the difference between this value and the median 3D RMSE of the $T_{\mathrm{M}}=10 \mathrm{~s}$ plus $T_{\mathrm{C}}=10 \mathrm{~s}$ combination was $<0.4 \mathrm{~mm}$. No significant differences were apparent among the RMSEs for $T_{\mathrm{M}}=70$ or the other $T_{\mathrm{M}} \mathrm{S}$, except in the case of $T_{\mathrm{M}}=4 \mathrm{D}-\mathrm{CBCT}$. Based on the population of patients and results of the inter-scan estimation, a combination of $T_{M}=10 \mathrm{~s}$ and $T_{\mathrm{C}}=10 \mathrm{~s}$ would be appropriate.

Our method is independent of the beam delivery technique and is widely applicable. Although no additional imaging is needed during beam irradiation, baseline drift correction, applied between fields or arcs, may be required in some cases. Thomas et al. investigated 

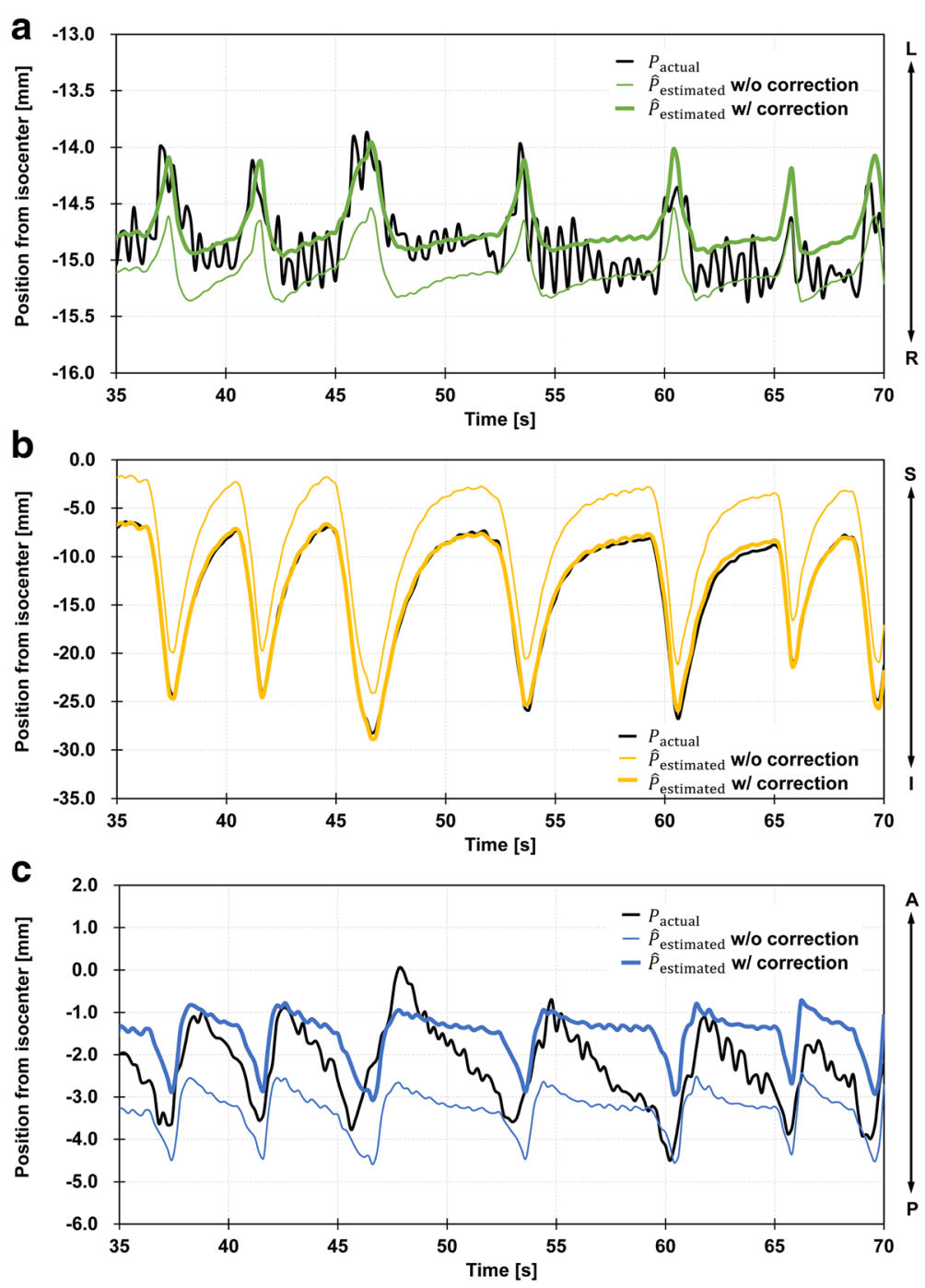

Fig. 7 Actual $\left(P_{\text {actual }}\right)$ and estimated target positions $\left(\hat{P}_{\text {estimated }}\right)$ in the (a) left-right $(\mathrm{LR})$, (b) superior-inferior $(\mathrm{SI})$, and (c) anterior-posterior (AP) directions with and without the baseline drift correction of the first scan of Patient 2, estimated from the estimation equation modeled by the data of the third scan. Both the modeling and correction periods was $10 \mathrm{~s}$

treatment times, including the time from the start of the first field or arc to the cessation of the last [26]. The treatment time of patients undergoing lung SBRT via flattening filter-associated intensity-modulated radiotherapy was $~ 15 \mathrm{~min}$. In such cases, baseline drift correction may be essential; the median elapsed time in this study was $10.1 \mathrm{~min}$. However, during flattening filterfree volumetric-modulated arc therapy, the treatment time can be reduced to $<3.3 \mathrm{~min}$. Thus, baseline drift correction may not be necessary with a short treatment time.

One limitation of this study was the additional imaging dose. The imaging dose delivered to a $2-\mathrm{cm}^{3}$ volume of skin during a 70 -s dual-source $4 \mathrm{D}-\mathrm{CBCT}$ scan was 7.4-10.5 cGy [27]. The imaging dose delivered by our method during the 10-s training period was approximately one-seventh of that value. However, if abdominal motion is captured during 3DCBCT performed to ensure appropriate patient positioning, projection data can also be used to model the estimation equation; thus, in the absence of baseline drift correction, no additional imaging doses would be required.

\section{Conclusions}

We developed a widely applicable method to estimate 3D target positions using an external respiratory surrogate without additional imaging during beam irradiation, and optimized training periods for modeling and baseline drift correction by reference to clinical data. The developed method is independent of beam delivery technique. We revealed that a long training 


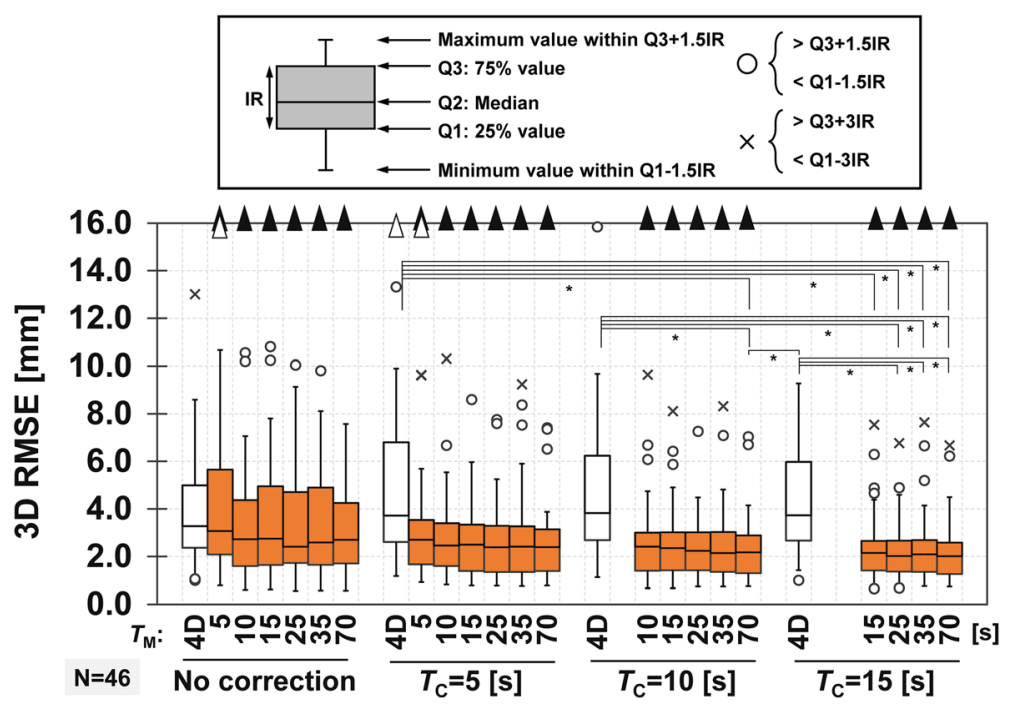

Fig. 8 Boxplots of the 3D RMSEs for each training period in terms of the modeling session $\left(T_{M}\right)$ and baseline drift correction $\left(T_{C}\right)$. White triangles: outliers out of range. Black triangles: outliers observed in patient included apnea and hyperpnea data. ${ }^{*} p<0.05$

period was not necessary; the optimal training period to model the estimation equation and baseline drift correction was determined to be $10 \mathrm{~s}$, to include more than one respiratory cycle for most patients in this study.

\section{Additional file}

Additional file 1: Dataset supporting our findings. (XLSX $1259 \mathrm{~kb})$

\section{Abbreviations}

2D: Two-dimensional; 3D: Three-dimensional; 4D: Four-dimensional; AP: Anterior-posterior; CBCT: Cone-beam computed tomography;

CT: Computed tomography; IR: Infrared reflective; KV: Kilovoltage; LR: Left-right; MV: Megavoltage; RMSE: Root-mean-square error;

SBRT: Stereotactic body radiotherapy; SI: Superior-inferior

\section{Funding}

This research was supported, in part, by the Practical Research for Innovative Cancer Control (No. 17ck0106303h0001) of Japan Agency for Medical Research and Development (AMED), and the Japan Society for the Promotion of Science (JSPS) Grant-in-Aid for JSPS Fellows (No. 16J08928).

\section{Availability of data and materials}

The dataset supporting the conclusions of this article is included within (Additional file 1).

\section{Authors' contributions}

HI developed and validated the method, performed the statistical analysis, and drafted the manuscript. MN, YI, TM, YM and TM conceived the study, and participated in its design and coordination, and helped draft the manuscript. IK helped to draft the manuscript. All authors have read and approved the final manuscript.

\section{Authors' information}

Hiraku Iramina: Research Fellow of the Japan Society for the Promotion of Science.

\section{Ethics approval and consent to participate}

This study followed all dictates of the Declaration of Helsinki. The Ethical Review Board of Kyoto University Hospital and Faculty of Medicine approved the research (approval number C862). Written consent to participate was obtained from each patient.

\section{Consent for publication}

Written consent was obtained from all patients for publication of this report and any accompanying images.

\section{Competing interests}

The authors declare that they have no competing interests.

\section{Publisher's Note}

Springer Nature remains neutral with regard to jurisdictional claims in published maps and institutional affiliations.

\section{Author details}

'Department of Nuclear Engineering, Graduate School of Engineering, Kyoto University, Nishikyo-ku, Kyoto 615-8530, Japan. ${ }^{2}$ Department of Radiation Oncology and Image-applied Therapy, Graduate School of Medicine, Kyoto University, 54 Kawahara-cho, Shogoin, Sakyo-ku, Kyoto 606-8507, Japan. ${ }^{3}$ Division of Medical Physics, Department of Information Technology and Medical Engineering, Human Health Sciences, Graduate School of Medicine, Kyoto University, 53 Kawahara-cho, Shogoin, Sakyo-ku, Kyoto 606-8507, Japan.

Received: 22 December 2017 Accepted: 5 April 2018

Published online: 19 April 2018

\section{References}

1. Iramina $H$, Nakamura M, lizuka $Y$, Mitsuyoshi T, Matsuo $Y$, et al. The accuracy of extracted target motion trajectories in four-dimensional cone-beam computed tomography for lung cancer patients. Radiother Oncol. 2016:121(1):46-51.

2. Jones BL, Westerly $\mathrm{D}$, Miften M. Calculating tumor trajectory and dose-ofthe-day using cone-beam CT projections. Med Phys. 2015:42(2):694-702.

3. Takahashi W, Yamashita H, Kida S, Masutani Y, Sakumi A, Ohtomo K, et al. Verification of planning target volume settings in volumetric modulated arc therapy for stereotactic body radiation therapy by using in-treatment 4dimensional cone beam computed tomography. Int J Radiat Oncol Biol Phys. 2012;86(3):426-31. 
4. Caillet V, Keall PJ, Colvill E, Hardcastle N, O'Brien R, Szymura K, et al. MLC tracking for lung SABR reduces planning target volumes and dose to organs at risk. Radiother Oncol. 2017;124(1):18-24.

5. Ehrbar S, Schmid S, Jöhl A, Klöck S, Guckenberger M, Riesterer O, et al. Validation of dynamic treatment-couch tracking for prostate SBRT. Med Phys. 2017:44(6):2466-77.

6. Toftegaard J, Hansen R, Ravkilde T, Macek K, Poulsen PR. An experimentally validated couch and MLC tracking simulator used to investigate hybrid couch-MLC tracking. Med Phys. 2017;44(3):798-809.

7. Takamiya M, Nakamura M, Akimoto M, Ueki N, Yamada M, Tanabe H, et al. Multivariate analysis for the estimation of target localization error in fiducial marker-based radiotherapy. Med Phys. 2016;43(4):1907-12.

8. Poulsen PR, Cho B, Langen K, Kupelian P, Keal PJ. Three-dimensional prostate position estimation with a single $x$-ray imager utilizing the spatial probability density. Phys Med Biol. 2008:53(16):4331-53.

9. Poulsen PR, Cho B, Keall PJ. A method to estimate mean position, motion magnitude, motion correlation, and trajectory of a tumor from cone-beam CT projections for image-guided radiotherapy. Int J Radiat Oncol Biol Phys. 2008;72(5):1587-96.

10. Hugo GD, Liang J, Yan D. Marker-free lung tumor trajectory estimation from a cone beam CT sonogram. Phys Med Biol. 2010;55(9):2637-50.

11. Li R, Fahimian BP, Xing LA. Bayesian approach to real-time 3D tumor localization via monoscopic $x$-ray imaging during treatment delivery. Med Phys. 2011;38(7):4205-14

12. Shieh CC, Keall PJ, Kuncic Z, Huang CY, Feain I. Markerless tumor tracking using short kilovoltage imaging arcs for lung image-guided radiotherapy. Phys Med Biol. 2015;60(8):9437-54.

13. Cho B, Suh Y, Dieterich S, Keall PJ. A monoscopic method for real-time tumour tracking using combined occasional $x$-ray imaging and continuous respiratory monitoring. Phys Med Biol. 2008;53(11):2837-55.

14. Cho B, Poulsen PR, Keall PJ. Real-time tumor tracking using sequential kV imaging combined with respiratory monitoring: a general framework applicable to commonly used IGRT systems. Phys Med Biol. 2010;55(12):3299-316.

15. Cho B, Poulsen PR, Ruan D, Sawant A, Keall PJ. Experimental investigation of a general real-time $3 \mathrm{D}$ target localisation method using sequential $\mathrm{kV}$ imaging combined with respiratory monitoring. Phys Med Biol. 2012;57(22):7395-407.

16. Akimoto $M$, Nakamura M, Mukumoto N, Yamada M, Ueki N, Matsuo Y, et al. Optimization of the $\mathrm{x}$-ray monitoring angle for creating a correlation model between internal and external respiratory signals. Med. Phys. 2012;39(10): 6309-15.

17. Bahig H, Campeau MP, Vu T, Doucet R, Nadeau DB, Fortin B, et al. Predictive parameters of CyberKnife fiducial-less (XSight lung) applicability for treatment of early non-small cell lung cancer: a single-center experience. Int J Radiat Oncol Biol Phys. 2013;87(3):583-9.

18. Fassi A, Schaerer J, Fernandes M, Riboldi M, Sarrut D, Baroni G. Tumor tracking method based on a deformable 4D CT breathing motion model driven by an external surface surrogate. Int J Radiat Oncol Biol Phys. 2014; 88(1):182-8

19. Akimoto M, Nakamura M, Mukumoto N, Tanabe H, Yamada M, Matsuo Y, et al. Predictive uncertainty in infrared marker-based dynamic tumor tracking with Vero4DRT. Med Phys 2013;40(9):091705-1-8.

20. Akimoto M, Nakamura M, Mukumoto N, Yamada M, Tanabe H, Ueki N, et al. Baseline drift correction of a correlation model for improving the prediction accuracy of infrared marker-based dynamic tumor tracking. J Appl Clin Med Phys. 2015;16(2):14-22.

21. Seppenwoolde Y, Shirato H, Kitamura K, Shimizu S, van Herk M, Lebesque $\mathrm{J}$, et al. Precise and real-time measurement of 3D tumor motion in lung due to breathing and heartbeat, measured during radiotherapy. Int J Radiat Oncol Biol Phys. 2002;53(4):822-34.

22. Ionascu D, Jiang SB, Nishioka S, Shirato H, Berbeco Rl. Internal-external correlation investigations of respiratory induced motion of lung tumors. Med Phys. 2007;34(10):3893-903.

23. Ruan D, Fessler JA, Batler JM, Keall PJ. Real-time profiling of respiratory motion: baseline drift, frequency variation and fundamental pattern change. Phys Med Biol. 2009;54(15):4777-92.

24. Ruan D, Fessler JA, Balter JM, Berbeco RI, Nishioka S, Shirato H. Inference of hysteretic respiratory tumor motion from external surrogates: a state augmentation approach. Phys Med Biol. 2008;53(11):2923-36.

25. Suh Y, Dieterich S, Cho B, Keal PJ. An analysis of thoracic and abdominal tumour motion for stereotactic body radiotherapy patients. Phys Med Biol. 2008;53(13):3623-40
26. Thomas EM, Popple RA, Predergast BM, Clark GM, Dobelbower MC, Fiveash JB. Effects of flattening filter-free and volumetric-modulated arc therapy delivery on treatment efficiency. J Appl Clin Med Phys. 2013;14(6):155-66.

27. Nakamura $M$, Ishihara $Y$, Matsuo $Y$, lizuka $Y$, Ueki $N$, Iramina $H$, et al. Quantification of the $\mathrm{kV} x$-ray imaging dose during real-time tumor tracking and from three- and four-dimensional cone-beam computed tomography in lung cancer patients with Monte Carlo simulation. J Radiat Res 2018: in press.

\section{Ready to submit your research? Choose BMC and benefit from:}

- fast, convenient online submission

- thorough peer review by experienced researchers in your field

- rapid publication on acceptance

- support for research data, including large and complex data types

- gold Open Access which fosters wider collaboration and increased citations

- maximum visibility for your research: over $100 \mathrm{M}$ website views per year

At BMC, research is always in progress.

Learn more biomedcentral.com/submissions 